\title{
Locked Nucleic Acid Molecular Beacons
}

\author{
Lin Wang ${ }^{1}$, Chaoyong James Yang ${ }^{1}$, Colin D. Medley ${ }^{1}$, \\ Steven A. Benner ${ }^{1,2^{*}}$ and Weihong Tan ${ }^{1 *}$ \\ ${ }^{1}$ Center for Research at Bio/nano Interface, Department of Chemistry, UF \\ Genetics Institute and McKnight Brain Institute, and ${ }^{2}$ Anatomy and Cell \\ Biology, University of Florida, Gainesville, FL, 32611-7200, USA
}

RECEIVED DATE (automatically inserted by publisher); E-mail: tan@ chem.ufl.edu, benner@chem.ufl.edu

\section{$\underline{\text { Supporting Information }}$}

\section{Sequences of MBs and target DNA}

6-mer stem MB: Cy3-CCTAGCTCTAAATCACTATGGTCGCGCTAGG-DABCYL 3-mer stem MB: $\quad$ Cy3-AGCTCTAAATCACTATGGTCGCGCT-DABCYL Complementary target: AGATTTAGTGATACCAGCG Single base mismatched target: AGATTTAGCGATACCAGCG

\section{Nuclease resistance comparison of both MBs}

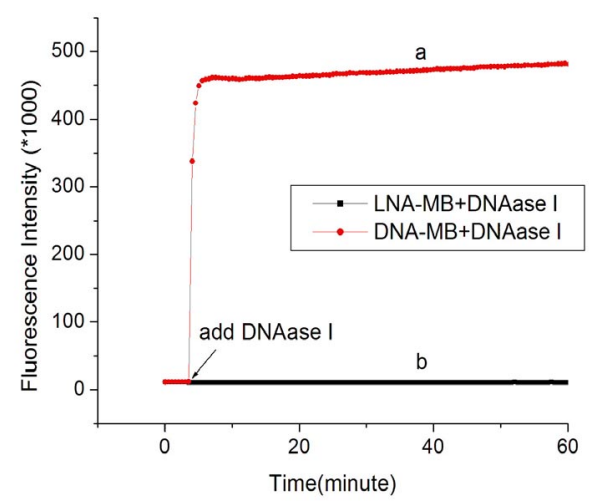

Figure 1. A comparison between the response of $100 \mathrm{nM}$ of (a) DNA-MB and (b) LNA-MB to the addition of $30 \mu \mathrm{g} / \mathrm{ml}$ DNAase I endonuclease. 


\section{Cellular experimental procedures}

Fluorescence imaging was conducted with a confocal microscope setup consisting of an Olympus IX-81 inverted microscope with an Olympus Fluoview 500 confocal scanning system. The cellular images were taken with a 40x 1.35 NA oil immersion objective. MDA-MB-231 breast carcinoma cells (American Type Culture Collection, Manassas, VA) were maintained in Dulbecco's Modification of Eagle's Medium (DMEM, Fisher Scientific) with 10\% fetal bovine serum (Invitrogen, Carlesbad, CA) and Gentamycin (Sigma, St. Louis, MO) in a $37^{\circ} \mathrm{C}$ cell culture incubator in $5 \% \mathrm{CO}_{2}$. A Leiden microincubator with a TC-202A temperature controller was used to keep the cells at $37^{\circ} \mathrm{C}$ during injection and monitoring. An Eppendorf Femtojet microinjector with $0.5 \mu \mathrm{m}$ Femtotips was used to inject the MBs $[1.0 \mu \mathrm{M}$ in $20 \mathrm{mM}$ Tris, $50 \mathrm{mM} \mathrm{NaCl}$ and $5 \mathrm{mM}$ $\mathrm{MgCl}_{2}$ buffer] along with reference probe [Alexa Fluor 488 (Molecular Probes, Eugene, OR)] into the cells to enable proper focusing and ratiometric analysis. After microinjection of the MBs, images for the monitoring experiments were taken every minute including an initial image for 60 minutes after a brief period during which the instrument was focused to yield the highest intensity for the reference probe. Images for the background comparison were taken immediately afterwards. Following this experiment each beacon was hybridized with excess target for 24 hours and subsequently microinjected into a cell to illustrate both the open and closed beacon signal. The images were assigned color representations and are not indicative of the actual emission wavelengths. The images were analyzed using the Fluoview 500 analysis software.



Figure 2. Images of both $\mathrm{MBs}$ with or without target inside of a MDA-MB-231 breast carcinoma cell (red) and the signal from the reference probe (orange) shows the outline of the cell. (A) LNA-MB without target (B) DNA-MB without target (C) LNA$\mathrm{MB}$ with target (D) DNA-MB with target. 